\title{
TWINSOL: A DUAL SUPERCONDUCTING SOLENOID SYSTEM FOR LOW-ENERGY RADIOACTIVE NUCLEAR BEAM RESEARCH*
}

\author{
M.Y. Lee, F.D. Becchetti, J.M. Holmes(a), T.W. O'Donnell, \\ M.A. Ratajczak, D.A. Roberts, J.A. Zimmerman ${ }^{(b)}$
}

Department of Physics, The University of Michigan, Ann Arbor, MI 48109-1120 USA

\author{
J.J. Kolata, L.O. Lamm, J. von Schwarzenberg, M. Wiescher \\ Department of Physics, University of Notre Dame, Notre Dame, IN 46556 USA
}

\begin{abstract}
A unique type of apparatus is currently under construction as part of an upgrade to the radioactive ion beam facility at the University of Notre Dame Nuclear Structure Laboratory. The device will consist of a pair of large in-line superconducting solenoids $\left(\mathrm{B}_{\mathrm{O}}=6\right.$ tesla, bore $\left.=30 \mathrm{~cm}\right)$ which will be used to produce, collect, transport, focus and analyze both stable and radioactive nuclear beams. This apparatus in conjunction with the recently upgraded accelerators at Notre Dame is especially well suited for the production and utilization of intense $(v i z .>106 / \mathrm{sec})$, low-energy $(1-10 \mathrm{MeV} / \mathrm{u})$, stable and radioactive nuclear beams relevant to the study of reactions involved in astrophysical processes. These improvements will allow for the production of radioactive beams of greater intensity, higher purity and at both higher and lower energies than previously available at this facility. The first phase of construction and results of initial tests will be reported.
\end{abstract}

\section{INTRODUCTION}

In the last decade many nuclear physics laboratories have successfully utilized various methods to produce and use radioactive nuclear beams (RNBs). A number of facilities have recently either upgraded their RNB capabilities or announced plans to do so.(1) Although the majority of these facilities are using or developing Isotopic Separation OnLine (ISOL) methods of RNB production, a few facilities, like the UM-UND RNB apparatus at the University of Notre Dame Nuclear Structure Laboratory (UND), have produced usable secondary radioactive beams by collecting reaction products from the bombardment of a primary beam upon specially selected targets. In contrast with other labs such as NSCL and GANIL that use a similar method (projectile fragmentation), but at much higher kinetic energies, UND is in a unique position to study low-energy nuclear reactions, especially those that are relevant to astrophysical processes.

A new device, "TwinSol", is currently being installed as a joint UM-UND project funded by NSF, UND and UM to extend the successful RNB research program at UND which began nine years ago.(2) This apparatus is an adaptation of the original concept of a dual solenoid spectrometer at IPNOrsay, France, by J.P. Schapira et al. $(3,4)$ The design and development of TwinSol is based on the previous experiences of our group in using superconducting solenoids to produce and utilize $\operatorname{RNBs}(5,6,7,8,9)$ which included the installations of a $3.5 \mathrm{~T}$ magnet at $\operatorname{UND}(10,11,12)$ and a $7 \mathrm{~T}$ solenoid at NSCL.(13,14) Thus TwinSol represents the third generation of superconducting solenoidal RNB research systems developed by our group.

\section{DESIGN}

TwinSol is built around two, in-line, large-bore, simple (no yoke or end compensation) custom designed superconducting solenoids spaced approximately 4 meters apart in the West Target Room experimental area of the UND Nuclear Structure Lab.(c) Primary beams are accelerated from a SNICS source through a $10 \mathrm{MV}$ FNTandem Van de Graaff. The basic setup is shown in Fig. 1.

Each solenoid is rated for a maximum central field strength of 6 tesla at a current of 100 amperes with an axial field integral of $\int \mathrm{Bdl}=3.8 \mathrm{~T} \mathrm{~m}$. The cryostats are ca. $98 \mathrm{~cm}$ in length with a $30 \mathrm{~cm}$ diameter warm bore which is slightly larger than the outer diameter of the beamline pipe. Since the magnets are mounted on rails, they can slide along the support structure independently of the other beamline components provided they do not come into contact with chambers or vacuum tees. In most instances the centerline of the first solenoid will be about $1.1 \mathrm{~m}$ from the production target, whereas the second solenoid position will vary depending upon the specific experiment and mode of operation. Typically the large magnetic forces between the two solenoids will prevent us from placing them closer than about $2 \mathrm{~m}$ apart from each other.

* Additional information may be found on the World Wide Web. The HTML document may be accessed at: http://www.physics.lsa.umich.edu/twinsol.

a) Permanent address: Physics Department, University of Texas, Austin, TX 78712

b) Permanent address: Department of Chemistry, University of Michigan, Ann Arbor, MI 48109-1055.

c) The solenoids were manufactured by Cryomagnetics, Inc., 1006 Alvin Weinberg, Dr. Oak Ridge, TN 37830. 


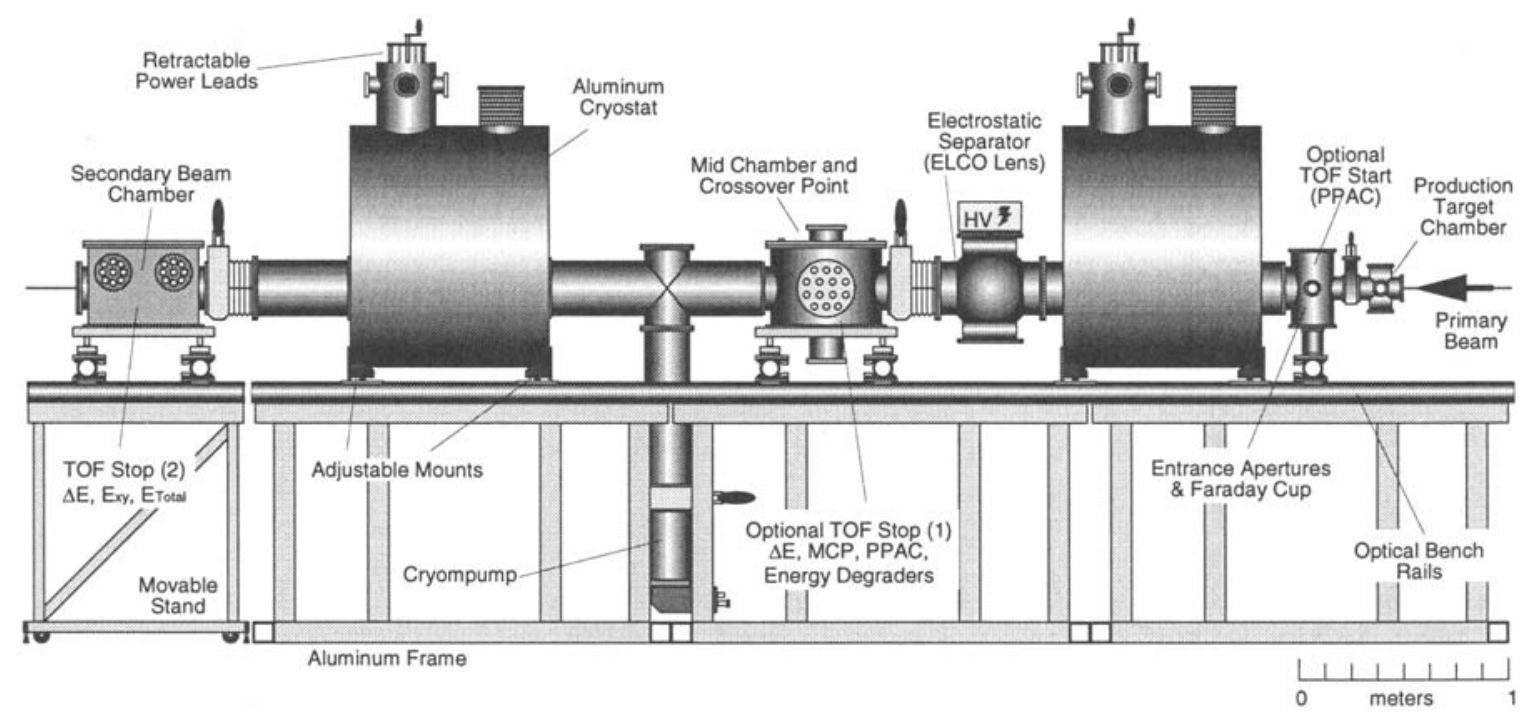

FIGURE 1. Scale illustration of the University of Michigan - University of Notre Dame double solenoid ion-optical RNB system, TwinSol.

Each magnet consists of ten concentrically wound coils of multi-filament $\mathrm{NbTi}$ wire that spliced together form a solenoid of length ca. $60 \mathrm{~cm}$ with inner and outer diameters of ca. $18 \mathrm{~cm}$ and $21 \mathrm{~cm}$ respectively. The coils are aligned to $\pm 1 \mathrm{~mm}$ and suspended inside the aluminum cryostat by a series of thin fiberglass and stainless steel supports that are designed to optimize the balance between physical strength characteristics and thermal insulation properties. The axes of the coils are offset below the axes of the cryostats such that $70 \%$ of the liquid helium (LHe) contained in the 290liter capacity cryostat will be above the coils (see Fig. 2). In fact, the bottom of the inner wall of the cryostat is less than $2.25 \mathrm{~cm}$ away from the bottom of the outside edge of the coil. The LHe cryostat is insulated with superinsulation, an $80^{\circ} \mathrm{K}$ liquid nitrogen shield and an inner helium vapor-cooled $20^{\circ} \mathrm{K}$ radiation shield.

The offset-bore design, the special retractable power lead/persistent heater switch system, and the unique supports allow for a low helium loss rate cryostat. Initial tests with the first solenoid demonstrated a LHe boil off rate of less than 0.17 liters per hour with leads retracted. Thus, optimistically we can run for about 40 days with a persistent current in the magnets without refilling helium. This is essential as there is no LHe liquifier or recovery system available and therefore LHe costs must be minimized.

The large-bore all-aluminum cryostats provide a relatively lightweight system that unlike stainless steel is also less susceptible to long-lived neutron activation. Wherever possible, we have also used aluminum components for the various cryostat and beamline subsystems. Besides the possibility of activation however, neutrons may also cause the decay of the magnetic field in a persistent current superconductor. Recently, experimenters at GANIL reported significant neutron heating in their SISSI RNB production system. (15) Although UND operates at very much lower energies and hence lower neutron fluxes than GANIL, whether or not TwinSol will experience similar difficulties remains to be seen (provisions are available for running in non-persistent mode). One of the advantages of the combination of large-bore cryostat and small production target chamber is that the geometry allows for tight neutron shielding around the production target combined with a suitable setback, thus offering greater radiation protection for the coil.

The production chamber is a custom aluminum ISO-100 4-way cross separated from the ISO-200 aperture and Faraday cup chamber by a gate valve. In addition to conventional production targets, a rotating target and a newly developed gas target cell (1 - 10 atm) which was successfully implemented in a recent RNB experiment $(16)$ are available. Behind the first solenoid is an ISO-250 4-way cross that will serve as the housing for a radial electrostatic field electrode (ELCO lens)(17) that is being developed to further select reaction products exiting from the first solenoid (see below). The secondary beam chamber will be mounted on a movable stand. For certain experiments that require especially low background, e.g. RNB $\gamma$-spectroscopy, the secondary chamber can be placed in the next room, behind a $1.3 \mathrm{~m}$ thick concrete wall.

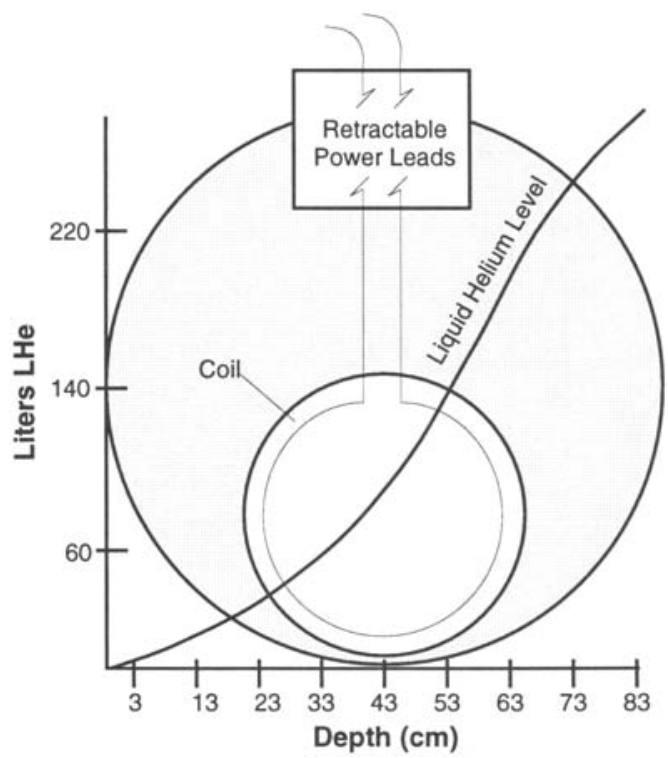

FIGURE 2. Offset bore and retractable power lead design help minimize the LHe loss rate. 


\section{MODES OF OPERATION}

There are two basic configurations available as shown in Fig. 3. The "parallel" flight mode is reserved for studying only the most magnetically rigid (high-mass, low chargestate) ions. The "crossover" mode offers several advantages over the parallel mode. First, because of the small area of the crossover point, better radiation shielding is available in the space between the two magnets.

Second, there are several useful devices which can be placed at the crossover point. Small area (thus low capacitance) detectors at the crossover focus will allow for fast timing of particles. Such a timing detector can be thin $(v i z .10 \mu \mathrm{m})$ silicon, a scintillator foil, or foil and microchannel plate. When detectors become count rate limited for high-intensity RNBs, then the intrinsic primary beam pulsing properties of the tandem accelerator will be used for timing. Beam pulses with good timing ( $\delta \mathrm{t} \sim 1 \mathrm{~ns}$ ) coupled with a typical flight time of $200 \mathrm{~ns}$ over the ca. $6 \mathrm{~m}$ flight path implies that $1 \%$ energy resolution is attainable, e.g. $200 \mathrm{keV}$ for a $20 \mathrm{MeV}$ RNB. This is sufficient to resolve most nuclear states.

Other possibilities include placing a position sensitive detector (PSD) near the crossover point to provide ray tracing information. Prototype work with the UM $7 \mathrm{~T}$ solenoid at NSCL has shown that a PPAC, even in a very high magnetic field, is usable as both an active "aperture" PSD and a timing detector.(13) In another configuration, unwanted reaction products that have the same rigidity as the secondary beam of interest and come to a focus at the crossover point may be filtered out by using a combination of apertures and "mid-plane absorbers" (energy loss foils) in conjunction with the second solenoid. In some instances the detector at the crossover point may also serve as the absorber. With the older $3.5 \mathrm{~T}$ solenoid RNB setup, ${ }^{8} \mathrm{Li}$ beams $\left(10^{8}\right.$ particles per sec.) of $80 \%$ purity were attainable at the focal plane. Using absorbers between the two solenoids in the new setup should provide up to $95 \%$ beam purity after the second magnet. In general, however, particle identification from time-of-flight information and the use of position sensitive detectors as active apertures will allow us to "separate" out the impurities during the data analysis.(13) These methods will be tested once the second solenoid is operational (late 1996).

Notice in Fig. 3 that the magnetic fields of the two solenoids are shown as parallel. An anti-parallel field configuration where the two magnets are opposing each other will still focus the beam as it passes through the two solenoids. Since solenoids are approximately double focusing - they focus particles as they enter the bore and then also focus as the particles leave - the difference in the modes is that anti-parallel field modes (due to the superposition of opposing fields in the central region) will have slightly reduced bending powers, hence longer crossover focal lengths, compared to parallel field modes (this has been verified using orbit-simulation codes). However, when certain detectors (PMTs/MCPs) are present between the two magnets, we may deliberately choose the anti-parallel configuration in order to reduce the field in the central region. Another device that is currently being developed is a high voltage electrostatic filter to be used to separate secondary beams. This consists of two concentric, cylindrically-shaped electrodes placed in between

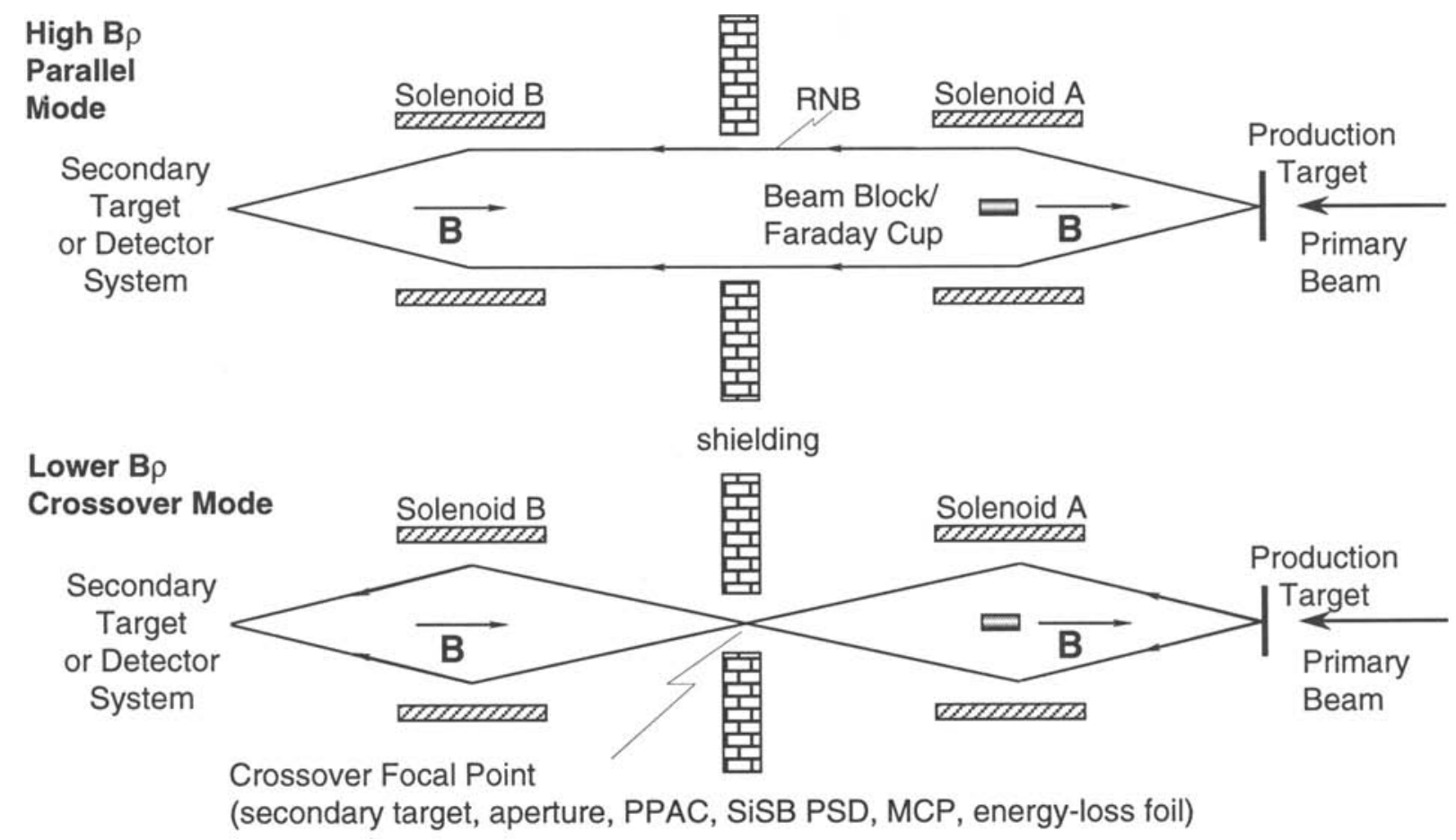

FIGURE 3. Parallel and crossover modes of operation. 
the two magnets. Simulations show that commercially available, modest (a few tens of $\mathrm{kV}$ ) power supplies will sufficiently separate the sort of low-energy RNBs that we will be producing. Figure 4 shows trajectories for a scenario of two helium isotopes with the same rigidity at several different exit angles from the production target. The background ${ }^{4} \mathrm{He}^{++}$would typically overwhelm the ${ }^{6} \mathrm{He}^{++}$of interest at the focal point. Initially, both follow identical paths through the solenoid but are separated by the electrostatic filter. The mean change in focal lengths for this particular simulation is $50 \mathrm{~cm}$. These calculations indicate that the effects of the radial electric field are most useful only if a small range of production angles $\left(\delta \theta \sim 2^{\circ}\right)$ is selected or ray-tracing is utilized. This method is therefore best suited only with certain RNBs and solenoid modes.

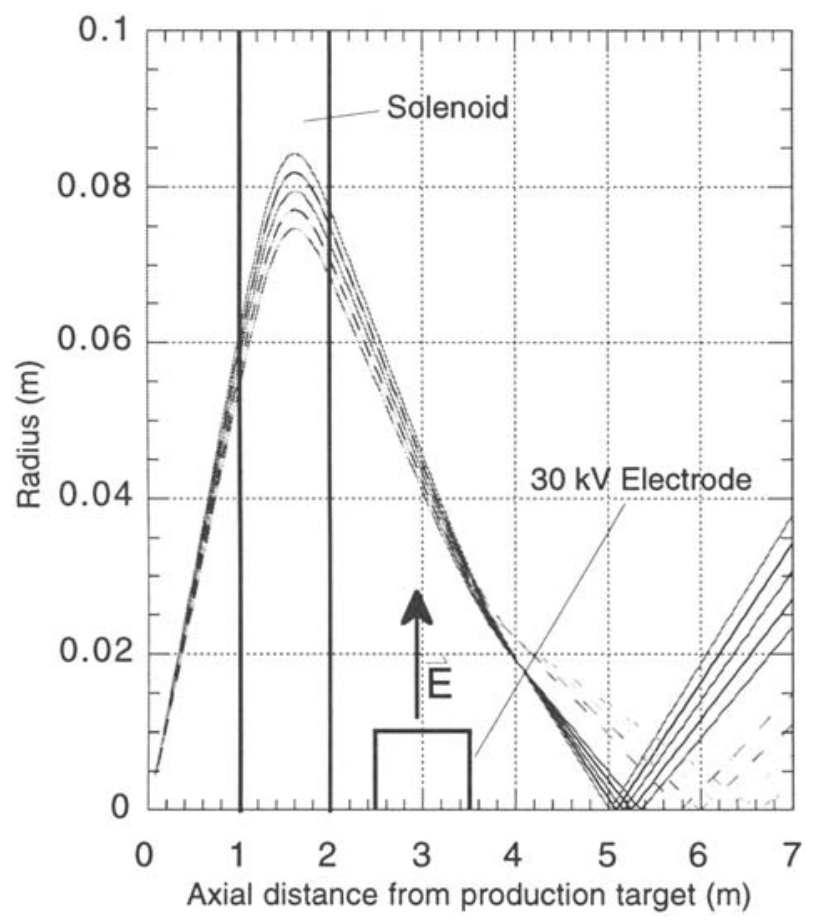

FIGURE 4. Calculated trajectories of ${ }^{4} \mathrm{He}$ and ${ }^{6} \mathrm{He}$ at $30 \mathrm{MeV}$ and 20 $\mathrm{MeV}$ respectively with exit angles between $3.0^{\circ}$ and $3.5^{\circ}$. The inner electrode has radius $1 \mathrm{~cm}$, length $1 \mathrm{~m}$, and electric potential at $30 \mathrm{kV}$. The grounded outer electrode is off scale in this plot (radius $=0.15 \mathrm{~m}$ ). Typical potentials will range from 30 to $80 \mathrm{kV}$.

\section{DISCUSSION AND CONCLUSIONS}

Although TwinSol is a major part of the upgrade to the UND Nuclear Structure Laboratory, other improvements at UND include the recent installation of a small KN Van de Graaff (for low-energy stable beam astrophysics research), a new helium ion source and on-going work with the FNTandem which has successfully delivered beams at a 10.4 MV terminal voltage. As such, TwinSol will allow for experiments with RNBs at higher energies (E/A $\rightarrow 8$ $\mathrm{MeV} / \mathrm{u}$ ) than previously studied at UND with the older, single UM 3.5 $\mathrm{T}$ solenoid ( $\mathrm{E} / \mathrm{A}<3 \mathrm{MeV} / \mathrm{u}$ ).
TwinSol will be especially well suited to the study of reactions involving both stable and radioactive light heavy ions $(\mathrm{A}<40)$ near the Coulomb barrier where there is unique and interesting physics. We anticipate that TwinSol will be fully operational by late 1996. As of October 1996, the first half of the apparatus, up to the mid chamber shown in Fig. 1, has been put in place. Updated information may be found on our WWW page.

\section{ACKNOWLEDGMENTS}

TwinSol is funded jointly by the University of Michigan, University of Notre Dame and the NSF through the Academic Research Infrastructure program, grant PHY9512199.

\section{REFERENCES}

1. Proc. Fourth International Conference on Radioactive Nuclear Beams, Omiya, Japan, June 4-7, 1996, Amsterdam, North-Holland, in press.

2. Becchetti, F.D., "LOW-ENERGY RADIOACTIVE BEAM EXPERIMENTS USING THE UM-UND SOLENOID RNB APPARATUS AT THE UND TANDEM: PAST, PRESENT AND FUTURE," presented at 14th Int'l Conf. on the Applications of Accelerators in Research and Industry, Denton, TX, Nov. 6-9, 1996, (this conference).

3. Schapira, J.P., Gales, S., and Laurent, H., "UN SPECTROMETRE CONSTITUE D'UN OU DE DEUX SOLENOIDES CRYOGENIQUES COAXIAUX," IPN-Orsay Report IPNO-PhN$7921,1979$.

4. Schapira, J.P., Azaiez, F., Fortier, S., Gales, S. Hourani, E., Kumpulainen, J., Maison, J.M., Nucl. Instrum. Methods, 224, 337-46, 1984.

5. Kolata, J.J., Morsad, A., Kong, X.J., Warner, R.E., Becchetti, F.D., Liu, W.Z., Roberts, D.A., Jänecke, J.W., Nucl. Instrum. Methods, B40/41, 503-6, 1989.

6. Becchetti, F.D., Liu, W.Z., Roberts, D.A., Jänecke, J.W., Kolata, J.J., Morsad, A., Kong, X.J., Warner, R.E., Phys. Rev. C, 40, R1104, 1989.

7. Smith, R.J. Kolata, J.J., Lamkin, K., Morsad, A., Ashktorab, K., Becchetti, F.D., Brown, J., Jänecke, J.W., Liu, W.Z., Roberts, D.A. Nucl. Instrum. Methods, A294, 26, 1990.

8. Becchetti, F.D., Brown, J.A., Ashktorab, K., Jänecke, J.W., Liu, W.Z., Roberts, D.A., Smith, R.J., Kolata, J.J., Lamkin, K., Morsad, A. Warner, R.E., Nucl. Instrum. Methods, B56/57, 554-8, 1991.

9. Becchetti, F.D., Liu, W.Z., Ashktorab, K., Bajema, J.F., Brown, J.A., Jänecke, J.W., Roberts, D.A., Kolata, J.J., Lamkin, K.L., Morsad, A., Smith, R.J., Kong, X.J., Warner, R.E., Phys. Rev. C, 48, 308-18, 1993.

10. Stern, R.L., Becchetti, F.D., Casey, T., Jänecke, J.W., Lister, P.M., Liu, W.Z., Kovar, D.G., Janssens, R.V.F., Vineyard, M.F., Phillips, W.R., Kolata, J., Rev. Sci. Instrum., 58, 1682, 1987.

11. Stern, R.L. "Design and Utilization of an Air-Core SuperconductingSolenoid Nuclear-Reaction-Product Spectrometer," Ph.D. Thesis, University of Michigan, UM-NP-RS-87-1, 1987

12. Liu, W.Z., "Production and use of Radioactive Ion Beams for Measurements of Nuclear Reactions," Ph.D. Thesis, University of Michigan, 1990.

13. O'Donnell, T.W., Aldredge, E., Beccheti, F.D., Brown, J.A., Conlan, P., Jänecke, J., Raymond, R.S., Roberts, D.A., Tickle, R.S., Griffin, H.C. Staynoff, J., Ronningen, R., Nucl. Instrum. Methods, A353, 215-6, 1994.

14. O'Donnell, T.W., Ph.D. Thesis, University of Michigan, in progress, 1997.

15. Savalle, A., Baelde, J.L., Baron, E., Berthe, C., Gillet, J., Grunbrerg, C., Jamet, C., Lemaitre, M., Moscatello, M.H., Ozille, M., "THE SISSI FACILITY AT GANIL," GANIL Report A96.02, 1996.

16. von Schwarzenberg, J., Kolata, J.J., Peterson, D., Santi, P., Belbot, M., Hinnefeld, J.D., Phys. Rev. C, 53, R2598, 1996.

17. Liu, W.Z., Becchetti, F.D., Stern, R., Rev. Sci. Instrum., 58, 220-2, 1987, and references cited there. 